\title{
Callus induction and plant regeneration of Valeriana officinalis are affected by different leaf explants and various concentrations of plant growth regulators
}

\author{
AKRAM Zamini ${ }^{1}$, ARASh MoKhtari ${ }^{2 *}$, Minoo TANSAZ ${ }^{3}$, MohSEn Zarei ${ }^{2}$ \\ ${ }^{1}$ Department of Agricultural Biotechnology, Payam Noor University of Isfahan, Isfahan, Iran \\ ${ }^{2}$ Agricultural Biotechnology Research Institute of Iran, Agricultural Research, \\ Education and Extension Organization (AREEO), Tehran, Iran \\ ${ }^{3}$ Department of Agricultural Biotechnology, Payam Noor University of Karaj, Karaj, Iran
}

\begin{abstract}
Valeriana officinalis, commonly known as valerian, is native to Europe and Asia and has economic importance as a medicinal plant. The effects of auxins (2,4-D and NAA) on callus induction were studied in vitro using basal piece of leaf + petiole (L1) and middle piece of leaf (L2) explants of $V$. officinalis. The best callus formation (95.83\%) was reached on MS containing 2,4-D $\left(1.5 \mathrm{mg} / \mathrm{l}^{-1}\right)+\mathrm{KIN}\left(1 \mathrm{mg} / \mathrm{l}^{-1}\right)$ although there was no significant difference with 2,4-D $\left(2 \mathrm{mg} / \mathrm{l}^{-1}\right)+\mathrm{KIN}\left(0.5\right.$ or $\left.1 \mathrm{mg} / \mathrm{l}^{-1}\right)$ on L2 explants. All calli successfully developed roots on callus induction media, a feature that can be used in the future for a large-scale production, saving time and cost. Among cytokinins (BAP, TDZ, and KIN) tested for callus differentiation, the highest regeneration (62.5\%), shoot number (5.87) and shoot length $(5.96 \mathrm{~cm})$, occurred on a combination of BAP $\left(0.5 \mathrm{mg} / \mathrm{l}^{-1}\right)+\operatorname{IBA}\left(0.5 \mathrm{mg} / \mathrm{l}^{-1}\right)$. The results revealed that the effect of explant type on callogenesis was also significant. In contrast to KIN or TDZ, addition of IBA to BAP showed positive synergistic effect for plant regeneration and shoot number per callus.
\end{abstract}

Key words: callogenesis; plant growth regulators; shoot regeneration; valerian
Abbreviations
BAP - 6-benzylaminopurine
IBA - indole-3-butyric acid
NAA - naphthalene acetic acid
KIN - kinetin
PGRs - plant growth regulators
MS - Murashige and Skoog medium
TDZ - thidiazuron
2,4-D - 2,4-dichlorophenoxyacetic acid

\section{Introduction}

The family Valerianaceae includes 400 species of which about 200 belong to Valeriana genus. Valeriana officinalis, commonly known as valerian, is native to Europe and Asia and has economic importance as a medicinal plant (Hidalgo et al., 2010, Patocka et al., 2010). Roots or rhizomes are the main parts of the plant that are medicinally used. Now, valerian extracts are considered as dietary supplements, which are formulated into tablets or soft gelatin capsules (Patocka et al., 2010), commonly used as anti-HIV, sleep aid, antidepressant, and antihypertensive agents (Ebrahimzadeh et al., 2008).
More than 150 chemical constituents including valerenic acids and valepotriates are found in valerian extract, which are often regarded as active ingredients (EkhteraeiTousi et al., 2010). Valerian is traditionally propagated through seeds or root divisions. Fresh seeds are only $30 \%$ viable, and this number goes down after the first year of storage. Root divisions also suffer from root rot and white mold infections (Rao et al., 2005). Besides, chemical constituents in valerian plants vary depending on different sources and growing conditions, processing methods and storage conditions (Patocka et al., 2010).

\footnotetext{
* Corresponding author: Agricultural Biotechnology Research Institute of Iran, Agricultural Research, Education and Extension Organization (AREEO), Tehran, Iran; e-mail: rshmokhtari@yahoo.com
} 
Therefore, developing a method for mass propagation of this valuable medicinal plant is essential to improve the quality and quantity of raw plant material to guarantee its commercial production. Callus induction provides a basis for future studies on genetic improvement, production of secondary metabolites through cell cultures, and regeneration of clonal plantlets through micropropagation of valerian. It is well established that callus induction can be used as an effective and applicable method for micropropagation of plant material. Mokhtari et al. (2015) have already successfully reported the callus induction and plant regeneration of medicinal plant Viola odorata. To evaluate the potential of hairy root production of Verbena officinalis under in vitro conditions, the regeneration by indirect organogenesis was also conducted (Zebarjadi et al., 2011). Previously, plant regeneration has been reported from callus and suspension cultures of Valeriana edulis (Castillo et al., 2000) and Valeriana wallichii (Mathur, 1991).

In in vitro cultures, plant growth regulators (PGRs) are important components playing key roles in the developmental processes. The auxin and cytokinin phytohormones interact to regulate many plant growth and developmental processes such as callus induction, root formation, growth of buds, and shoot formation (Mokhtari et al., 2015).

The aim of the present study was to evaluate the effect of auxins $(2,4-\mathrm{D} / \mathrm{NAA})$ on callus induction and cytokinins $(\mathrm{BAP} / \mathrm{TDZ} / \mathrm{KIN})$ on complete plant regeneration of various leaf-derived explants from $V$. officinalis $\mathrm{L}$.

\section{Materials and methods}

\section{Plant material}

Mature valerian seeds were collected from the Research Center for Agriculture and Natural Resources, Isfahan, Iran, during the growing seasons in 2012 and 2013. Disinfection was carried out according to the following scheme:

a) washing with running tap water five times,

b) soaking in $0.1 \% \mathrm{HgCl}_{2}$ solution for 10 minutes,

c) submerging in $70 \%$ ethanol for $60 \mathrm{~s}$,

d) mixing with $2 \% \mathrm{NaClO}+0.05 \%$ Tween -20 for $10 \mathrm{~min}$,

e) rinsing thrice with sterile distilled water under aseptic conditions.

For germination, the disinfected seeds were sown on solid MS medium (Murashige and Skoog, 1962) supple- mented with $3 \%$ sucrose and without growth regulators. All cultures were kept at $24 \pm 2^{\circ} \mathrm{C}$, photoperiod of $16 \mathrm{~h}$ light, with an intensity of 3000 lux illuminated with fluorescent tubes.

\section{Callus induction}

The young leaves of in vitro seed-derived plantlets were dissected to basal piece $+0.5 \mathrm{~cm}$ of its petiole (L1) and middle piece of about $0.5 \times 0.5 \mathrm{~cm}$ (L2). Six explants were wounded with scalpel and transferred into Petri plates containing MS basal medium supplemented with all treatments of possible combinations of 2,4-D or NAA $\left(0,0.5,1,1.5,2,2.5\right.$, and $\left.3 \mathrm{mg} / \mathrm{l}^{-1}\right)$ with $\mathrm{KIN}(0,0.5$ and $\left.1 \mathrm{mg} / \mathrm{l}^{-1}\right)$ in two separate experiments. In one experiment, the combinations of 2,4-D and KIN were investigated (designated as $\mathrm{C} 1$ ), and in the other experiment, the possible combinations of NAA and KIN were studied (designated as $\mathrm{C} 2$ ). Each treatment was repeated 4 times. Cultures were maintained for 4 weeks in dark at 25 $\pm 1^{\circ} \mathrm{C}$. After 30 -days incubation period, data were recorded on the percentage of callus induction. The well-formed calli were broken into $\sim 0.5 \mathrm{~cm}^{3}$ pieces and subcultured on the same medium for further proliferation.

\section{Shoot regeneration from callus}

The basal medium used for all regeneration experiments was MS mineral formulation variously supplemented with BAP, TDZ, or KIN $\left(0,0.5,1,1.5\right.$, and $\left.2 \mathrm{mg} / 1^{-1}\right)$ in combination with $\operatorname{IBA}\left(0,0.5\right.$, and $\left.1 \mathrm{mg} / \mathrm{l}^{-1}\right)$. The possible combinations of BAP * IBA are listed in Table 3 and designated as R1; also, the combinations of TDZ * IBA and combinations of $\mathrm{KIN}{ }^{*}$ IBA are designated as R2 and R3, respectively (Table 3 ). The proliferated calli were dissected into small pieces and five of them were transferred to culture bottles containing regeneration treatments. All cultures were incubated in a growth room at $22 \pm 2{ }^{\circ} \mathrm{C}$ with photoperiod of $16 \mathrm{~h}$ light (photosynthetic photo flux density; PPFD $=40-45 \mu \mathrm{mol} \mathrm{m}^{2} \mathrm{~s}^{-1}$ ) provided by cool white fluorescent tubes. The frequency of regeneration (\%), the number of micro-shoots per regenerated callus, and the length of shoots for each treatment (which comprised 4 replicates) were recorded after 30-days incubation period.

\section{Plantlet acclimatization}

The best in vitro rooted plantlets were removed from the culture bottles, washed carefully with running 
Table 1. Mean squares (MS) of ANOVA based on FCRD for callus induction percentage of $V$. officinalis in MS medium by SAS ver. 9.1.

\begin{tabular}{|c|c|c|c|c|c|}
\hline \multicolumn{2}{|l|}{ Experiment $\mathrm{C} 1$} & & \multicolumn{3}{|c|}{ Experiment $\mathrm{C} 2$} \\
\hline Source & $\mathrm{df}$ & $\begin{array}{c}\text { Callus } \\
\text { induction\% }\end{array}$ & Source & df & $\begin{array}{c}\text { Callus } \\
\text { induction } \%\end{array}$ \\
\hline explants & 1 & $3201.989^{* *}$ & explants & 1 & $2210.345^{\text {** }}$ \\
\hline $2,4-\mathrm{D}$ & 6 & $19106.385^{* *}$ & NAA & 6 & $2277.768^{* *}$ \\
\hline $\mathrm{KIN}$ & 2 & $42.450^{\mathrm{ns}}$ & KIN & 2 & $11.570^{\mathrm{ns}}$ \\
\hline explants * 2,4-D & 6 & $103.087^{\mathrm{ns}}$ & explants * NAA & 6 & $30.315^{\mathrm{ns}}$ \\
\hline explants * KIN & 2 & $21.517^{\mathrm{ns}}$ & explants * KIN & 2 & $1.647^{\mathrm{ns}}$ \\
\hline $2,4-\mathrm{D} * \mathrm{KIN}$ & 12 & $1317.499^{* *}$ & NAA * KIN & 12 & $2681.047^{* *}$ \\
\hline explants * 2,4-D * KIN & 12 & $42.708^{\mathrm{ns}}$ & explants * NAA * KIN & 12 & $40.237^{\mathrm{ns}}$ \\
\hline Error & 126 & 446.448 & Error & 126 & 610.636 \\
\hline Total & 167 & & Total & 167 & \\
\hline
\end{tabular}

** - significant at $P \leq 0.01, \mathrm{~ns}$ - not statistically significant, df - degrees of freedom
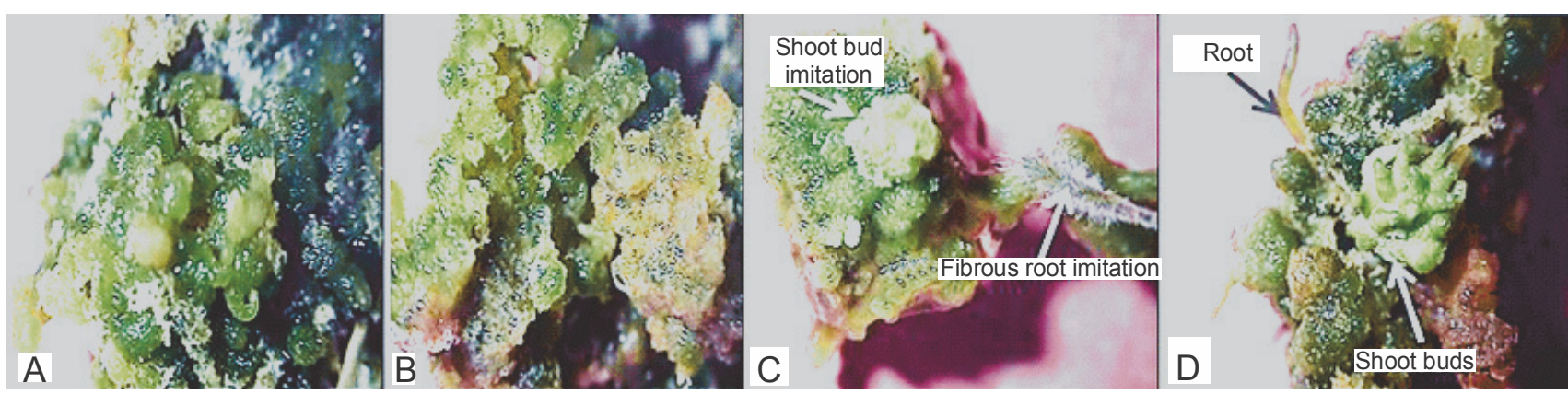

Fig. 1. Callus initiation: A and B - on a basal piece of leaf with petiole of $V$. officinalis after 3 weeks of growth in MS media containing 2,4-D $\left(1.5 \mathrm{mg} / \mathrm{l}^{-1}\right)+\mathrm{KIN}\left(1 \mathrm{mg} / \mathrm{l}^{-1}\right)$; $\mathrm{C}$ - initiation of green spots and $\mathrm{D}$ - shoot primordial

tap water to remove the remaining agar, and then were transplanted into plastic pots containing peat + cocopeat + perlite $(2: 1: 1)$ and covered with polyethylene bag to maintain the humidity. The covers were gradually perforated and plantlets acclimatized in a controlled greenhouse conditions at $24^{\circ} \mathrm{C}$ under $16 / 8 \mathrm{~h}$ (light/dark) photoperiod.

\section{Statistical analysis}

All experiments were set up as a factorial completely randomized design (FCRD). Significance was determined by the analysis of variance (ANOVA), and the mean differences were compared by LSD test at 0.05 probability level using the SAS computer program (ver. 9).

\section{Results}

Effect of 2,4-D, NAA, and explants on callus induction

Two experiments were separately designed for two types of explants $(\mathrm{L} 1, \mathrm{~L} 2)$ of $V$. officinalis, in order to investigate the effect of auxins (2,4-D and NAA) on callus induction. The analysis of variance for callus induction (\%) suggested significant differences $(P=0.01)$ for $2,4-\mathrm{D}$, explants, and two-way interaction among 2,4-D * KIN. However, two-way interaction among explants * 2,4-D, explants * $\mathrm{KIN}$, and three-way interaction between $2,4-\mathrm{D}$ * $\mathrm{KIN}$ * explants were insignificant (Table 1; experiment $\mathrm{C} 1$ ).

In most of the treatments of experiment 1 , callus initiation from explants was observed about 3 weeks after 
Table 2. Interaction effects of different explants and PGRs on \% callus induction of $V$. officinalis

\begin{tabular}{|c|c|c|c|}
\hline \multirow{2}{*}{\multicolumn{2}{|c|}{ Plant growth regulators $\left(\mathrm{mg} / \mathrm{l}^{-1}\right)$}} & \multicolumn{2}{|c|}{$\%$ Callus induction } \\
\hline & & \multirow{2}{*}{$\begin{array}{c}\text { Basal piece }+ \text { petiole }(\mathrm{L} 1) \\
0^{\mathrm{i}}\end{array}$} & \multirow{2}{*}{$\begin{array}{c}\text { Middle piece of leaf (L2) } \\
0^{\mathrm{i}}\end{array}$} \\
\hline \multirow{21}{*}{ 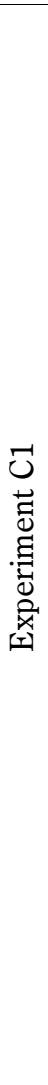 } & $2,4-\mathrm{D}(0)+\mathrm{KIN}(0)$ & & \\
\hline & 2,4-D (0) + KIN (0.5) & $0^{\mathrm{i}}$ & $0^{\mathrm{i}}$ \\
\hline & $2,4-\mathrm{D}(0)+\mathrm{KIN}(1)$ & $0^{\mathrm{i}}$ & $0^{\mathrm{i}}$ \\
\hline & $2,4-\mathrm{D}(0.5)+\mathrm{KIN}(0)$ & $37.49^{\text {gh }}$ & $45.83^{\mathrm{fg}}$ \\
\hline & $2,4-\mathrm{D}(0.5)+\mathrm{KIN}(0.5)$ & $54.16^{\mathrm{f}}$ & $62.49^{\mathrm{e}}$ \\
\hline & 2,4-D (0.5) + KIN (1) & $66.66^{\text {de }}$ & $73.42^{\text {cd }}$ \\
\hline & 2,4-D (1) + KIN (0) & $75.08^{\mathrm{c}}$ & $72.55^{\text {cd }}$ \\
\hline & 2,4-D (1) + KIN (0.5) & $58.33^{\mathrm{ef}}$ & $62.49^{\mathrm{e}}$ \\
\hline & 2,4-D (1) + KIN (1) & $54.16^{\mathrm{f}}$ & $62.49^{\mathrm{e}}$ \\
\hline & 2,4-D (1.5) + KIN (0) & $74.99^{\text {cd }}$ & $73.33^{\text {cd }}$ \\
\hline & 2,4-D (1.5) + KIN (0.5) & $49.99^{\mathrm{fg}}$ & $58.33^{\mathrm{f}}$ \\
\hline & $2,4-\mathrm{D}(1.5)+\mathrm{KIN}(1)$ & $74.30^{\text {cd }}$ & $95.83^{\mathrm{a}}$ \\
\hline & $2,4-\mathrm{D}(2)+\mathrm{KIN}(0)$ & $70.82^{\mathrm{de}}$ & $74.99^{\text {cd }}$ \\
\hline & $2,4-\mathrm{D}(2)+\mathrm{KIN}(0.5)$ & $73.30^{\text {cd }}$ & $94.33^{\mathrm{a}}$ \\
\hline & $2,4-\mathrm{D}(2)+\mathrm{KIN}(1)$ & $73.30^{\text {cd }}$ & $94.66^{\mathrm{a}}$ \\
\hline & $2,4-\mathrm{D}(2.5)+\mathrm{KIN}(0)$ & $70.82^{\text {de }}$ & $79.16^{\mathrm{c}}$ \\
\hline & $2,4-\mathrm{D}(2.5)+\mathrm{KIN}(0.5)$ & $70.83^{\mathrm{de}}$ & $87.49^{\mathrm{b}}$ \\
\hline & $2,4-\mathrm{D}(2.5)+\mathrm{KIN}(1)$ & $74.99^{\text {cd }}$ & $88.33^{\mathrm{b}}$ \\
\hline & $2,4-\mathrm{D}(3)+\mathrm{KIN}(0)$ & $54.16^{\mathrm{f}}$ & $66.66^{\text {de }}$ \\
\hline & $2,4-\mathrm{D}(3)+\mathrm{KIN}(0.5)$ & $33.33^{\mathrm{h}}$ & $41.66^{g}$ \\
\hline & 2,4-D (3) + KIN (1) & $66.66^{\mathrm{de}}$ & $79.16^{\mathrm{c}}$ \\
\hline \multirow{21}{*}{ 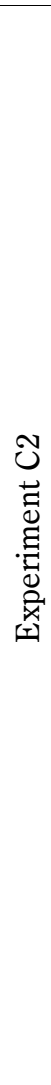 } & $\mathrm{NAA}(0)+\mathrm{KIN}(0)$ & $0^{f}$ & $0^{f}$ \\
\hline & NAA (0) + KIN (0.5) & $0^{\mathrm{f}}$ & $0^{\mathrm{f}}$ \\
\hline & NAA (0) + KIN (1) & $0^{f}$ & $0^{f}$ \\
\hline & NAA (0.5) + KIN (0) & $0^{f}$ & $0^{\mathrm{f}}$ \\
\hline & NAA (0.5) + KIN (0.5) & $0^{\mathrm{f}}$ & $0^{\mathrm{f}}$ \\
\hline & NAA (0.5) + KIN (1) & $0^{\mathrm{f}}$ & $0^{\mathrm{f}}$ \\
\hline & NAA (1) + KIN (0) & $0^{f}$ & $0^{f}$ \\
\hline & NAA (1) + KIN (0.5) & $0^{f}$ & $0^{\mathrm{f}}$ \\
\hline & $\mathrm{NAA}(1)+\mathrm{KIN}(1)$ & $0^{f}$ & $0^{f}$ \\
\hline & $\mathrm{NAA}(1.5)+\mathrm{KIN}(0)$ & $20.83^{\text {cd }}$ & $20.83^{\text {cd }}$ \\
\hline & NAA (1.5) + KIN (0.5) & $16.66^{\mathrm{d}}$ & $20.83^{\mathrm{cd}}$ \\
\hline & NAA (1.5) + KIN (1) & $20.83^{\text {cd }}$ & $23.33^{\mathrm{cd}}$ \\
\hline & NAA (2) + KIN (0) & $33.33^{\mathrm{b}}$ & $33.33^{\mathrm{c}}$ \\
\hline & $\mathrm{NAA}(2)+\mathrm{KIN}(0.5)$ & $8.33^{\mathrm{e}}$ & $26^{\mathrm{bc}}$ \\
\hline & NAA (2) + KIN (1) & $20.83^{\text {cd }}$ & $20.83^{\text {cd }}$ \\
\hline & NAA $(2.5)+$ KIN (0) & $29.43^{\mathrm{c}}$ & $30.00^{c}$ \\
\hline & NAA $(2.5)+$ KIN $(0.5)$ & $37.5^{\mathrm{b}}$ & $66.66^{\mathrm{a}}$ \\
\hline & NAA (2.5) + KIN (1) & $33.33^{b}$ & $54.16^{\mathrm{a}}$ \\
\hline & NAA (3) + KIN (0) & $0^{\mathrm{f}}$ & $0^{f}$ \\
\hline & NAA (3) + KIN (0.5) & $0^{\mathrm{f}}$ & $0^{f}$ \\
\hline & NAA (3) + KIN (1) & $0^{f}$ & $0^{f}$ \\
\hline
\end{tabular}



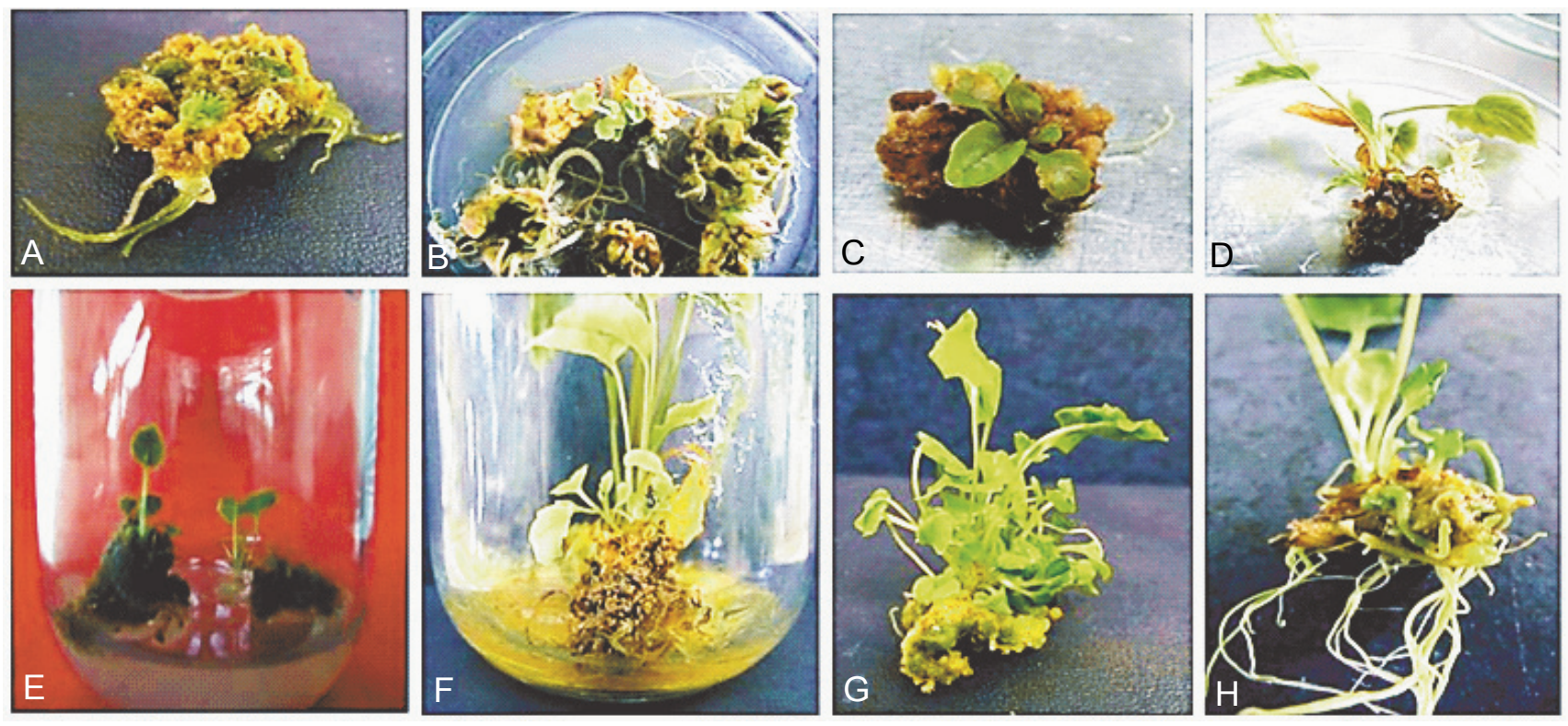

Fig. 2. A - Initiation of shoot primordial; B-G - regenerated micro-shoots with several leaves on BAP $\left(0.5 \mathrm{mg} / \mathrm{l}^{-1}\right)$ + IBA $\left(0.5 \mathrm{mg} / 1^{-1}\right) ; \mathrm{H}$ - dissected shoots with well-developed root system of $V$. officinalis

the beginning of incubation, and the best values for callus formation (95.83\%; Fig. 1A nad 1B) were reached on MS containing 2,4-D $\left(1.5 \mathrm{mg} / \mathrm{l}^{-1}\right)+\mathrm{KIN}\left(1 \mathrm{mg} / \mathrm{l}^{-1}\right)$. Callus induction occurred slightly less efficiently on 2,4-D $\left(2 \mathrm{mg} / \mathrm{l}^{-1}\right)$ in combination with $\mathrm{KIN}\left(0.5\right.$ or $\left.1 \mathrm{mg} / \mathrm{l}^{-1}\right)$ on L2 explants; statistical analysis revealed, however, that there was no significant difference when the two tested growth conditions were compared (Table 2). In experiment $\mathrm{C} 2$, callus induction was significantly $(P=0.01)$ influenced by the type of explants, NAA addition, and NAA*KIN interaction (Table 1). Irrespective of KIN and explants, no sign of callus induction was observed for NAA concentrations that were lower than $1.5 \mathrm{mg} \cdot \mathrm{l}^{-1}$ or higher than $2 \mathrm{mg} \cdot 1^{-1}$. Based on the results presented in Table 2, NAA was less effective on callus induction than 2,4 -D. The highest percentage $(66.66 \%)$ of produced calli occurred on L2 explants sown on NAA $\left(2.5 \mathrm{mg} / \mathrm{l}^{-1}\right)$ $+\mathrm{KIN}\left(0.5 \mathrm{mg} / \mathrm{l}^{-1}\right)$, but again the statistical analysis revealed no significant difference for the combination of NAA $\left(2.5 \mathrm{mg} / \mathrm{l}^{-1}\right)+\mathrm{KIN}\left(1 \mathrm{mg} / \mathrm{l}^{-1}\right)$, and also for NAA $\left(2.5 \mathrm{mg} / \mathrm{l}^{-1}\right)$ in combination with $\mathrm{KIN}\left(0.5\right.$ or $\left.1 \mathrm{mg} / \mathrm{l}^{-1}\right)$ on L2 explants. In experiments $\mathrm{C} 1$ and $\mathrm{C} 2$, and irrespective of the type of explants, auxin (2, 4-D/NAA) was essential for callogenesis as callus induction responses were not observed when MS medium was fortified with KIN alone. There was no callus induction in PGRs free media (Table 2).
All formed calli developed fibrous roots on callus induction media (especially in media containing NAA), and therefore there was no need to transfer the explants into the rhizogenesis medium (Fig. $1 \mathrm{C}$ and $1 \mathrm{D}$ ).

\section{Effect of BAP, KIN, and TDZ on shoot regeneration}

Of about 14-20 days after transferring the proliferated calli to shoot regeneration media (R1, R2 and R3), in some treatments, green spots were observed on some of the surfaces of calli (Fig. 1C), which then converted into primordial shoots (Fig. 1D; Fig. 2A) and then after another 1-2 weeks into micro-shoots with several leaves (Fig. 2B-D). Each regeneration medium was individually evaluated, and the results are summarized in Table 3. In R1 medium, the highest values for regeneration percentage (82.5\%; Fig. 2E), number of shoots (5.87, Fig. $2 \mathrm{~F}$ and $2 \mathrm{G})$, and the length of shoots $(5.96 \mathrm{~cm})$ occurred on a medium treated with $\operatorname{BAP}\left(0.5 \mathrm{mg} / \mathrm{l}^{-1}\right)+\mathrm{IBA}$ $\left(0.5 \mathrm{mg} / \mathrm{l}^{-1}\right)$. Although regeneration was also observed on BAP alone, the percentage of regeneration reached the maximum value only when IBA was added (Table 3 ). According to the results for $\mathrm{R} 2$ media and irrespective of concentrations of IBA, MS containing TDZ ( 0.5 or $1 \mathrm{mg} / \mathrm{l}^{-1}$ ) showed the maximum percentage of differentiated calli and number/length of micro-shoots (Table 3 ). Only one concentration of KIN $\left(0.5 \mathrm{mg} / \mathrm{l}^{-1}\right)$ alone or combined with IBA $\left(0.5 \mathrm{mg} / \mathrm{l}^{-1}\right)$ showed regeneration 
Table 3. Effects of BAP, TDZ, and KIN on regeneration percentage, number and length of callus-derived micro-shoots of $V$. officinalis

\begin{tabular}{|c|c|c|c|c|}
\hline Media & PGRs (mg/ $\left.\mathrm{l}^{-1}\right)$ & \% Regeneration & $\begin{array}{l}\text { Number } \\
\text { of shoots }\end{array}$ & $\begin{array}{l}\text { Length } \\
\text { of shoots }\end{array}$ \\
\hline \multirow{18}{*}{$\mathrm{R} 1$} & $\mathrm{BAP}(0)+\mathrm{IBA}(0)$ & $0^{c}$ & $0^{c}$ & $0^{c}$ \\
\hline & $\mathrm{BAP}(0)+\operatorname{IBA}(0.5)$ & $0^{c}$ & $0^{\mathrm{c}}$ & $0^{c}$ \\
\hline & $\mathrm{BAP}(0)+\mathrm{IBA}(1)$ & $0^{c}$ & $0^{c}$ & $0^{c}$ \\
\hline & $\operatorname{BAP}(0.5)+\operatorname{IBA}(0)$ & $0^{c}$ & $0^{c}$ & $0^{c}$ \\
\hline & $\operatorname{BAP}(0.5)+\operatorname{IBA}(0.5)$ & $82.5^{\mathrm{a}}$ & $5.87^{\mathrm{a}}$ & $5.96^{\mathrm{a}}$ \\
\hline & $\mathrm{BAP}(0.5)+\mathrm{IBA}(1)$ & $45^{\mathrm{b}}$ & $2.12^{\mathrm{b}}$ & $1.25^{\mathrm{b}}$ \\
\hline & $\mathrm{BAP}(1)+\operatorname{IBA}(0)$ & $35^{\mathrm{b}}$ & $2^{\mathrm{b}}$ & $1.03^{\mathrm{bc}}$ \\
\hline & $\mathrm{BAP}(1)+\operatorname{IBA}(0.5)$ & $45^{\mathrm{b}}$ & $1.87^{\mathrm{b}}$ & $1.77^{\mathrm{b}}$ \\
\hline & $\mathrm{BAP}(1)+\mathrm{IBA}(1)$ & $0^{c}$ & $0^{c}$ & $0^{c}$ \\
\hline & $\mathrm{BAP}(1.5)+\mathrm{IBA}(0)$ & $0^{c}$ & $0^{\mathrm{c}}$ & $0^{c}$ \\
\hline & $\operatorname{BAP}(1.5)+\operatorname{IBA}(0.5)$ & $0^{c}$ & $0^{c}$ & $0^{\mathrm{c}}$ \\
\hline & $\mathrm{BAP}(1.5)+\mathrm{IBA}(1)$ & $0^{c}$ & $0^{c}$ & $0^{c}$ \\
\hline & $\mathrm{BAP}(2)+\operatorname{IBA}(0)$ & $0^{c}$ & $0^{c}$ & $0^{c}$ \\
\hline & $\mathrm{BAP}(2)+\operatorname{IBA}(0.5)$ & $0^{\mathrm{c}}$ & $0^{\mathrm{c}}$ & $0^{\mathrm{c}}$ \\
\hline & $\mathrm{BAP}(2)+\mathrm{IBA}(1)$ & $0^{\mathrm{c}}$ & $0^{\mathrm{c}}$ & $0^{\mathrm{c}}$ \\
\hline & $\mathrm{BAP}(2.5)+\mathrm{IBA}(0)$ & $0^{c}$ & $0^{c}$ & $0^{c}$ \\
\hline & $\operatorname{BAP}(2.5)+\operatorname{IBA}(0.5)$ & $0^{c}$ & $0^{\mathrm{c}}$ & $0^{\mathrm{c}}$ \\
\hline & $\mathrm{BAP}(2.5)+\mathrm{IBA}(1)$ & $0^{c}$ & $0^{c}$ & $0^{c}$ \\
\hline \multirow{18}{*}{$\mathrm{R} 2$} & $\mathrm{TDZ}(0)+\mathrm{IBA}(0)$ & $0^{\mathrm{c}}$ & $0^{c}$ & $0^{\mathrm{b}}$ \\
\hline & $\mathrm{TDZ}(0)+\operatorname{IBA}(0.5)$ & $0^{c}$ & $0^{\mathrm{c}}$ & $0^{\mathrm{b}}$ \\
\hline & TDZ (0) + IBA (1) & $0^{\mathrm{c}}$ & $0^{c}$ & $0^{\mathrm{b}}$ \\
\hline & $\mathrm{TDZ}(0.5)+\mathrm{IBA}(0)$ & $0^{c}$ & $0^{c}$ & $0^{\mathrm{b}}$ \\
\hline & $\operatorname{TDZ}(0.5)+\operatorname{IBA}(0.5)$ & $68.5^{\mathrm{a}}$ & $3.12^{\mathrm{a}}$ & $2.39^{\mathrm{a}}$ \\
\hline & $\operatorname{TDZ}(0.5)+\operatorname{IBA}(1)$ & $16.25^{\mathrm{c}}$ & $0.31 b^{c}$ & $0.09^{\mathrm{e}}$ \\
\hline & $\mathrm{TDZ}(1)+\mathrm{IBA}(0)$ & $62.5^{\mathrm{a}}$ & $3.62^{\mathrm{a}}$ & $3.06^{\mathrm{a}}$ \\
\hline & $\mathrm{TDZ}(1)+\mathrm{IBA}(0.5)$ & $25^{\mathrm{b}}$ & $1.25^{\mathrm{b}}$ & $0.63^{\mathrm{b}}$ \\
\hline & TDZ (1) + IBA (1) & $43.75^{\mathrm{a}}$ & $3.12^{\mathrm{a}}$ & $2.39^{\mathrm{a}}$ \\
\hline & $\mathrm{TDZ}(1.5)+\mathrm{IBA}(0)$ & $0^{c}$ & $0^{c}$ & $0^{\mathrm{b}}$ \\
\hline & TDZ (1.5) + IBA (0.5) & $0^{c}$ & $0^{c}$ & $0^{\mathrm{b}}$ \\
\hline & $\mathrm{TDZ}(1.5)+\mathrm{IBA}(1)$ & $0^{c}$ & $0^{c}$ & $0^{\mathrm{b}}$ \\
\hline & $\mathrm{TDZ}(2)+\operatorname{IBA}(0)$ & $0^{c}$ & $0^{c}$ & $0^{\mathrm{b}}$ \\
\hline & $\mathrm{TDZ}(2)+\operatorname{IBA}(0.5)$ & $0^{c}$ & $0^{c}$ & $0^{\mathrm{b}}$ \\
\hline & TDZ (2) + IBA (1) & $0^{c}$ & $0 \mathrm{c}$ & $0^{\mathrm{b}}$ \\
\hline & $\mathrm{TDZ}(2.5)+\mathrm{IBA}(0)$ & $0^{c}$ & $0^{c}$ & $0^{\mathrm{b}}$ \\
\hline & $\operatorname{TDZ}(2.5)+\operatorname{IBA}(0.5)$ & $0^{c}$ & $0^{c}$ & $0^{\mathrm{b}}$ \\
\hline & $\mathrm{TDZ}(2.5)+\mathrm{IBA}(1)$ & $0^{c}$ & $0^{\mathrm{c}}$ & $0^{\mathrm{b}}$ \\
\hline
\end{tabular}


Table 3 continued

\begin{tabular}{|c|c|c|c|c|}
\hline Media & PGRs $\left(\mathrm{mg} / \mathrm{l}^{-1}\right)$ & \% Regeneration & $\begin{array}{l}\text { Number } \\
\text { of shoots }\end{array}$ & $\begin{array}{l}\text { Length } \\
\text { of shoots }\end{array}$ \\
\hline \multirow{18}{*}{ R3 } & $\mathrm{KIN}(0)+\operatorname{IBA}(0)$ & $0^{\mathrm{b}}$ & $0^{\mathrm{b}}$ & $0^{\mathrm{b}}$ \\
\hline & $\operatorname{KIN}(0)+\operatorname{IBA}(0.5)$ & $0^{\mathrm{b}}$ & $0^{\mathrm{b}}$ & $0^{\mathrm{b}}$ \\
\hline & $\mathrm{KIN}(0)+\mathrm{IBA}(1)$ & $0^{\mathrm{b}}$ & $0^{\mathrm{b}}$ & $0^{\mathrm{b}}$ \\
\hline & $\mathrm{KIN}(0.5)+\operatorname{IBA}(0)$ & $6.25^{\mathrm{b}}$ & $0.31^{\mathrm{b}}$ & $0.09^{b}$ \\
\hline & $\operatorname{KIN}(0.5)+\operatorname{IBA}(0.5)$ & $31.25^{\mathrm{a}}$ & $2.43^{\mathrm{a}}$ & $2.94^{\mathrm{a}}$ \\
\hline & $\operatorname{KIN}(0.5)+\operatorname{IBA}(1)$ & $0^{\mathrm{b}}$ & $0^{\mathrm{b}}$ & $0^{\mathrm{b}}$ \\
\hline & $\mathrm{KIN}(1)+\operatorname{IBA}(0)$ & $0^{\mathrm{b}}$ & $0^{\mathrm{b}}$ & $0^{\mathrm{b}}$ \\
\hline & $\mathrm{KIN}(1)+\operatorname{IBA}(0.5)$ & $0^{\mathrm{b}}$ & $0^{\mathrm{b}}$ & $0^{\mathrm{b}}$ \\
\hline & $\mathrm{KIN}(1)+\mathrm{IBA}(1)$ & $0^{\mathrm{b}}$ & $0^{\mathrm{b}}$ & $0^{\mathrm{b}}$ \\
\hline & $\operatorname{KIN}(1.5)+\operatorname{IBA}(0)$ & $0^{\mathrm{b}}$ & $0^{\mathrm{b}}$ & $0^{\mathrm{b}}$ \\
\hline & $\mathrm{KIN}(1.5)+\operatorname{IBA}(0.5)$ & $0^{\mathrm{b}}$ & $0^{\mathrm{b}}$ & $0^{\mathrm{b}}$ \\
\hline & $\mathrm{KIN}(1.5)+\operatorname{IBA}(1)$ & $0^{\mathrm{b}}$ & $0^{\mathrm{b}}$ & $0^{\mathrm{b}}$ \\
\hline & $\mathrm{KIN}(2)+\operatorname{IBA}(0)$ & $0^{\mathrm{b}}$ & $0^{\mathrm{b}}$ & $0^{\mathrm{b}}$ \\
\hline & $\mathrm{KIN}(2)+\operatorname{IBA}(0.5)$ & $0^{\mathrm{b}}$ & $0^{\mathrm{b}}$ & $0^{\mathrm{b}}$ \\
\hline & $\mathrm{KIN}(2)+\operatorname{IBA}(1)$ & $0^{\mathrm{b}}$ & $0^{\mathrm{b}}$ & $0^{\mathrm{b}}$ \\
\hline & $\mathrm{KIN}(2.5)+\mathrm{IBA}(0)$ & $0^{\mathrm{b}}$ & $0^{\mathrm{b}}$ & $0^{\mathrm{b}}$ \\
\hline & $\mathrm{KIN}(2.5)+\operatorname{IBA}(0.5)$ & $0^{\mathrm{b}}$ & $0^{\mathrm{b}}$ & $0^{\mathrm{b}}$ \\
\hline & $\mathrm{KIN}(2.5)+\mathrm{IBA}(1)$ & $0^{\mathrm{b}}$ & $0^{\mathrm{b}}$ & $0^{\mathrm{b}}$ \\
\hline
\end{tabular}

responses. The best percentage of regeneration and number/length of micro-shoots were reached on KIN $\left(0.5 \mathrm{mg} / \mathrm{l}^{-1}\right)$ with $31.25 \%, 2.43$ and $2.94 \mathrm{~cm}$, respectively.

As IBA was added to $\left(0.5 \mathrm{mg} / \mathrm{l}^{-1}\right) \mathrm{KIN}$, regeneration percentage significantly decreased from $31.25 \%$ to $6.25 \%$. In R1, R2, and R3 media, auxin (IBA) alone produced no regeneration responses (Table 3 ). Table 3 presents a positive correlation among callus differentiation capacity (regeneration \%) and the number and length of regenerated micro-shoots. On the contrary, the highest values of regeneration percentage were accompanied by the maximum number of shoots derived from appropriate callus. Dissected shoots with well-developed root system (Fig. 2H) were transplanted and transferred to a greenhouse, and all of them showed a normal growth (Fig. 3).

\section{Discussion}

Different plant tissues may have various levels of endogenous plant growth regulators (PGRs), and therefore the type of explants might have an important impact on the callus induction process (Das et al., 2012). Endogenous content of IAA in different segments of a leaf of

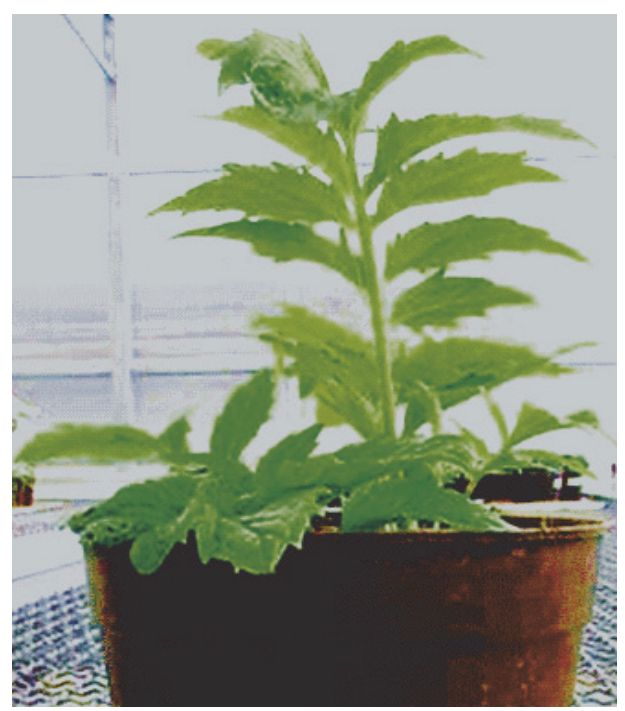

Fig. 3. Normal growth of in vitro-derived plantlet of $V$. officinalis under greenhouse conditions

Verbesina encelioides was studied, and it was demonstrated that a specific correlation existed among the segment and callus formation; the middle segment had higher level of IAA than apical and basal segments. It was observed that the young leaf of $V$. encelioides did 
not produce callus to the same extent as the middle segment of the leaf did in MS medium containing BAP + NAA/IAA and BAP without any addition of auxins (Karnawat et al., 2010). In our study, middle pieces of leaves (L2) showed higher callus induction capacity than basal pieces of leaves with its petioles (L1). The frequency of callogenesis varied in all the explants and applied PGRs. Application of 2,4-D/NAA was very effective on callus formation, especially when combined with a cytokinin (KIN). Between applied auxin combined with a cytokinin (KIN), 2,4-D produced the highest percentage of callus from L2 explants. This result agrees with previous study reporting that $2,4-\mathrm{D}$ was considered as one of the most effective auxins for the callus induction and effective in case of several different plants. For example, leaves, stems, and roots of in vitro plants were used as explants for indirect organogenesis of $V$. officinalis. According to Zebarjadi et al. (2011), the highest frequency of callus formation was achieved on a medium supplemented with $0.1 \mathrm{mg} / \mathrm{l}^{-1} \mathrm{KIN}+2 \mathrm{mg} / \mathrm{l}^{-1} 2$,4-D for leaf and root explants (100\%) as well as with $0.1 \mathrm{mg} / \mathrm{l}^{-1}$ $\mathrm{KIN}+10 \mathrm{mg} / \mathrm{l}^{-1} 2,4$-D for stem explants $(100 \%)$. The frequency of callus induction was found to be optimum in rhizome explants of Valeriana jatamansi on media supplemented with $0.5 \mathrm{mg} / \mathrm{l}^{-1} 2,4-\mathrm{D}$ (Das et al., 2012); 2,4-D was also effective in initiating callus in two types of explants (cotyledons and embryos) of soybean (Joyner et al., 2010). MS medium supplemented with 2,4-D (6.78 and $9.05 \mu \mathrm{m})$ alone and in combination with BAP $(6.66-$ $15.53 \mu \mathrm{m})$ or with KIN $(6.97-16.26 \mu \mathrm{m})$ induced callus in petiole explants of Viola serpens (Vishwakarma et al., 2013). In a study on callus induction from various explants in Vigna radiate, 2,4-D proved to be better than NAA, and an addition of KIN further enhanced the growth of callus (Rao et al., 2005). It has been previously shown that callogenesis and cell division were stimulated faster and better in rapeseed cultivars when 2,4-D was combined with a cytokinin (BA), NAA (with or without BA), however, stimulated root formation and rhizogenesis (Tavakkol Afshari et al., 2011).

Healthy calli derived from explants of $V$. oficinalis were used for shoot regeneration. Regeneration percentage of green shoot buds occurred variously on calli transferred to regeneration media containing cytokinins (BAP/KIN/NAA) in a combination with an auxin (IBA). From the results obtained in our investigation, a synergistic influence of cytokinin in combination with IBA was evident. The results showed that BAP stimulated higher shoot bud induction (82.5\%) in comparison with other cytokinins. The highest percentage of shoot regeneration was obtained on a medium containing $0.5 \mathrm{mg} / \mathrm{l}^{-1}$ $\mathrm{NAA}+2 \mathrm{mg} / \mathrm{l}^{-1} \mathrm{BAP}(60.00 \%)$ in leaf-derived calli of $V$. officinalis (Zebarjadi et al., 2011), which is lower than $62.5 \%$ of regenerated shoots induced (in the present study) by TDZ alone $\left(1 \mathrm{mg} / \mathrm{l}^{-1}\right)$. The superiority of BAP over KIN in multiple shoot induction was also reported in some of medicinal plants such as Viola serpens (Vishwakarma et al., 2013). Between different concentrations of BAP tested, $4 \mathrm{mg} / \mathrm{l}^{-1}$ was found highly effective in multiple shoot regeneration in calli of $V$. serpens and the average number of shoots ( $2.6 \mathrm{~cm}$ height) per explants raised under treatment up to $36.4 \mathrm{~cm}$. Kinetin was found less effective compared to BAP since after the fourth subsequent subculture the average number of shoots (1.7 $\mathrm{cm}$ height) was $22 \mathrm{~cm}$, on MS medium supplemented with $\left(2.5 \mathrm{mg} / \mathrm{l}^{-1}\right) \mathrm{KIN}$ (Vishwakarma et al., 2013). The efficiency of TDZ on shoot regeneration in many medicinal plants was reported. In comparison, among TDZ and KIN, MS medium supplemented with $0.75 \mathrm{mg} / \mathrm{l}^{-1} \mathrm{TDZ}$ in combination with $0.5 \mathrm{mg} / \mathrm{l}^{-1} \mathrm{NAA}$ showed the highest regeneration frequency $(88.6 \%)$ and produced the highest number of shoot buds (15.20 \pm 0.20 ) capable of growing into single plants of $V$. jatamansi. Our findings also confirmed the superiority of TDZ over KIN. Medium fortified with equal concentrations $\left(0.5 \mathrm{mg} / \mathrm{l}^{-1}\right)$ of TDZ and NAA produced 12.30 \pm 0.21 shoots having $80.4 \%$ regeneration potential (Das et al., 2012). Transferring the shoots into the rooting medium was not needed because the shoots were strongly rooted in the callus induction media. Similarly, $100 \%$ callus explants showed the development of fibrous roots on callus induction medium in $V$. serpens (Vishwakarma et al., 2013). Zebarjadi et al. (2011) reported an extra rooting medium for promoting roots in derived plantlets, but in the current study, the rooting medium was not needed; this inconsistency might be caused by the application of more types or higher concentrations of auxins (particularly NAA) or different types of explants used in our callogenesis experiments.

\section{Conclusions}

We describe a simple and efficient protocol for the propagation of valuable medicinal plant species Valeriana 
officinalis by indirect organogenesis system. From the results, we observe that callus induction clearly depends on the type of PGRs and the type of explants. The best results for callogenesis were obtained with middle piece of leaf as an explant grown on MS medium containing 2,4-D $\left(1.5-2 \mathrm{mg} / \mathrm{l}^{-1}\right)$ in combination with $\mathrm{KIN}\left(0.5-1 \mathrm{mg} / \mathrm{l}^{-1}\right)$.

For shoot regeneration, the data showed that the highest percentage of callus differentiation, number of shoots per callus, and shoot length were obtained in R1 medium supplemented with BAP $\left(0.5 \mathrm{mg} / \mathrm{l}^{-1}\right)+$ IBA $\left(0.5 \mathrm{mg} / \mathrm{l}^{-1}\right)$. This procedure circumvents the need to set up a rooting medium, and thus saves time and cost. The protocol could be beneficial for the large-scale production of disease-free, genetically uniform plant material and for the conservation of species. Also, callus production can be useful for the extraction of pharmaceutically important constituents on a commercial scale using bioreactor systems.

\section{References}

Castillo P., Judith M., Abrolam R., Georgina H., Migguel L. (2000) Plant regeneration from callus and suspension culture of Valerianaedulis spp. Procera via simultaneous organogenesis and somatic embryogenesis. Plant. Sci. 151: $115-119$.

Das J., Mao A.A., Handique P.J. (2012) Callus-mediated organogenesis and effect of growth regulators on production of different valepotriates in Indian valerian (Valeriana jatamansi Jones.). Acta Physiol. Plant. 35: 55-63.

Ebrahimzadeh H., Radjabian T., EkhteraieTousi S., Niknam V., Mozaffarian V. (2008) Evaluation of some Iranian wild species from valerianaceae as commerical sources of valepotriates. J. Biol. Sci. 8: 549-555.
EkhteraeiTousi S., Radjabian T., Ebrahimzadeh H., Niknam V. (2010) Enhanced production of valerenic acids and valepotriates by in vitro cultures of Valeriana officinalis $L$. Int. J. Plant. Prod. 4: 209-222.

Hidalgo O., elMathez J., Garcia S., Garnatje T., Pellicer J., Valles J. (2010) Genome size study in the valerianaceae: first results and new hypotheses. J. Bot. Volume 2010, Article ID 797246, 19 pages.

Joyner E.Y., Boykin L.S., Lodhi M.A. (2010) Callus induction and organogenesis in soybean [Glycine $\max (L) M e r r] c$.$v .$ Pyramid from mature cotyledons and embryos. Open Plant Sci. J. 4: 18-21.

Karnawat M., Jain D., Singh A., Malik C.P. (2010) In vitro plant regeneration from different leaf segments of Verbesina encelioides and correlation with endogenous level of IAA. Plant Tissue Cult. Biotech. 20: 195-201.

Mathur J., Ahuja P.S. (1991) Plant regeneration from suspension cultures of Valeriana wallichii. D.C. Plant Sci. 81: 111-116.

Mokhtari A., Otroshy M., Barekat T. (2015) Plant regeneration through callus induction on medicinal herb Viola odorata - role of plant growth regulator and explants. Agr. Forest 61: 191-170.

Rao S., Patil P., Kaviraj C.P. (2005) Callus induction and organogenesis from various explants in Vigna radiate $(L$. Wilczek. Indian Biotechnol. 4: 556-560.

Tavakkol Afshari R., Angoshtaril R., Kalantari S. (2011) Ef fects of light and different plant growth regulators on induction of callus growth in rapeseed (Brassica napus L.) genotypes. Plant OMICS 4: 60-67.

Vishwakarma U.R., Guray A.M., Sharma P.Ch. (2013) Regeneration of multiple shoots from petiole callus of Viola serpens Wall. Pharmacognosy Res. 5: 86-92.

Zebarjadi A.R., Najafi Sh., Ghasempour H.R., Motamedi J. (2011). Establishment of a practical tissue culture for producing hairy roots of Valeriana officinalis L. via Agrobacterium rhizogenes. J. Med. Plants Res. 5: 4984-4992. 